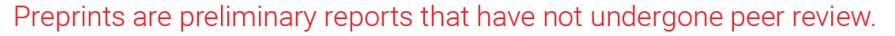 \\ They should not be considered conclusive, used to inform clinical practice, or referenced by the media as validated information. \\ Dimensions of sexual violence in perimenopausal women: A Systematic review protocol
}

\section{Mahboubeh Hajifoghaha}

Shiraz Medical School: Shiraz University of Medical Sciences

\section{Shiva Alizadeh}

Hormozgan University of Medical Sciences

\section{Samira Mokhlesi}

Azad University: Islamic Azad University https://orcid.org/0000-0002-1923-9455

Fatemeh Dabiri ( $\nabla$ fateme.dabiri@gmail.com )

\section{Study Protocol}

Keywords: Perimenopausal women, Sexual violence, Systematic review

Posted Date: February 22nd, 2021

DOI: https://doi.org/10.21203/rs.3.rs-54629/v2

License: (c) (1) This work is licensed under a Creative Commons Attribution 4.0 International License. Read Full License 


\section{Abstract}

\section{Background}

Violence against women is a crisis in public health and breaking human rights. Women who are sexually abused suffer a lot of physical, psychological and emotional injuries. Sexual violence has more adverse outcomes for middle-aged women, their families and society.

\section{Methods}

The present study will be conducted to review the dimensions of sexual violence in perimenopausal women. In this study, published articles from 2010 to 2020 will be reviewed. For this purpose, the articles that have the inclusion criteria will be selected by searching in databases PubMed, Scopus, Web of Science, ProQuest, SID and, Magiran using appropriate keywords. Articles of systematic review, letters to the editor, abstracts, and clinical trials will be excluded. A STROBE checklist will be used to assess the quality of articles.

\section{Discussion}

This systematic review will be addressed sexual violence in perimenopausal women as well as demographic factors affecting the existence of sexual violence. The results can be a guide for health planners to reduce this social problem and solve the worries of this group of society by providing the counseling programs.

Trial registration: The results of this study will be submitted to the journals a systematic article. Also, this protocol is registered in PROSPERO with number CRD42020191679.

\section{Background}

Sexual health is one of the sustainable development goals (SDG) until 2030. Sexual violence is one of the dimensions of sexual health [1]. Violence against women is an important issue of global health and a fundamental violation of human rights [2]. Regardless of economic, religious, or racial issues, 10 to 69 percent of women are exposed to violence during their lifetime that about 50 percent of them are accompanied by sexual violence.

Sexual violence includes any unusual or illegal acts of sexual intercourse without the consent of the person or humiliation and violence by the sexual partner during sexual intercourse. [4]. In sexual violence, there is usually no physical signs [5], but women who are abused by sexual violence suffer from psychological and emotional traumas and become disappointment and depressed about their sexual life [6]. In some cultures, forcible sexual intercourse between married people may not be considered rape, while psychologists consider it sexual violence and domestic sexual violence [7]. 
Perimenopause period includes the final years of a woman's reproductive period which begins with menstrual irregularities and amenorrhea after one year of amenorrhea. The postmenopausal stage which typically includes one-third of women's life exposes them to extensive physical and mental changes [8]. Menopausal symptoms are largely related to biological and hormonal changes associated with aging [9]. Decreasing estrogen in the early stages of menopause leads to a wide range of symptoms that can affect a woman's physical, mental and sexual health. Also, it affects social work and activities, mood, communication with others, and enjoyment of life and ultimately their quality of life [10].

The most important reasons of sexual disorders during this period include physiological changes in the body, psychological disorders and lack of sexual awareness. In this regard, many of the aggressions, disagreements, monitoring and control of couples in other's activities can be related to these disorders [11].

Studies show this health problem is common in the world. In a review study, $17 \%$ of all women who were the victim of recent sexual violence had 65 years or older [12]. The results of a study conducted in five countries reported the prevalence of sexual violence $3.6 \%$ in women aged 60 and over [13].

The World Health Organization has stated that menopausal women who have experienced sexual violence may change in their physiological response to stress. These changes are related to the hormonal and physiological status of menopause and aging and it can be exacerbated by trauma, so it may make her more sensitive to the symptoms of aging and menopause $[9,14]$. According to the sensitivity of menopause period and the increase in life expectancy, women's sexual health and its subsets, i.e., sexual violence, should be the main focus and care of this period because it causes irreversible difficulties. Due to the fact that the study of sexual violence of in perimenopausal women in different regions of the world has been reported sporadically and comprehensive information in this field is not available that it should consider this issue with a wider view and understand the extent of the problem and finally, it can be provided strategies for the prevention or intervention ratio to solve this health problem; Therefore, the researchers decided to conduct the present study to examine the dimensions of sexual violence against perimenopausal women to review and classify the findings of related articles on sexual violence of perimenopause in the world over the past decade.

\section{Methods}

This study is a systematic review of article will assess the sexual violence among perimenopausal women. To fulfill the present study, the electronic sources in English, including Medline / PubMed, Scopus, Web of Science, ProQuest and Embase will be systematically searched. Search term combinations will be perimenopause, middle age, midlife, climacteric, sexual violence, sexual assault, sexual abuse, sexual aggression, sexual harassment, sexual bullying, sexual coercion, sex offenses, spouse abuse, intimate partner violence, partner abuse, partner violence, intimate partner abuse. Which will combine using "OR" and "AND" operators. For each database, words and expressions will be chosen from a controlled vocabulary (MeSH, EMTREE, and others) and free-text searching. The search strategy 
will be designed by an information specialist. For completeness of the study, Persian language databases in such as SID and Magiran will be also searched with equivalent Persian keywords.

Two authors independently will be reviewed the titles and abstracts of the articles. If the subject matter seems to be relevant to aim of our study, the full article will be extracted and reviewed. Finally, all articles that meet the inclusion criteria will be selected.

- The inclusion criteria:

1. All articles have published from 2010 to Jan 31, 2020.

2. Persian and English articles published in Iranian and non-Iranian scientific journals.

3. Articles that had dealt with assessing the sexual violence among perimenopause women.

4. All observational studies, including descriptive, descriptive-analytic, case-control, and cohort studies published.

- The exclusion criteria:

We aim to identify original research articles assessing sexual violence in midlife (perimenopause) women. In this review, we define midlife women as those aged $\geq 45$ years. The exclusion criteria are repeated articles, papers not in English or Persian, papers in which the method and sample size had not been well specified, articles which had worked on only younger women, and papers that had measured the violence (not sexual violence) on the older women. Investigation of the inclusion and exclusion criteria of articles will be performed based on their title and abstract. After removing the papers not qualifying the inclusion criteria, all papers which are eligible are examined.

- Extracting the data:

To extract data from the text of the paper, two authors independently extract the information using the researcher-made form. This form includes the following cases:

1. General specifications (first author, year of publication, referee's name, article code, review date)

2. The purpose of conducting the study

3. Sample size and target group

4. Type of study (descriptive, descriptive-analytical, case control, group and longitudinal)

5. Collecting samples (sampling location and tool used)

6. Results 
The third author evaluates all extracted forms independently and, if disagreed with other authors, it is given to the fourth author.

- Primary and secondary outcomes:

Primary outcomes in this study are the rate of sexual violence in women in perimenopause age, the impact of sexual violence on the health of women in perimenopause, as well as demographic factors affecting the incidence of sexual violence in this age group which will be examined. Secondary outcomes will be included decreasing self-confidence, disclosure of violence and loss of moral and human dignity, reducing personal and social security and Suicidal thoughts

- Quality assessment:

In this study, a STROBE checklist will be used to evaluate the quality of articles. This checklist includes 22 various topics in observational studies. This checklist assesses the title and purpose of the research, population and participants, sampling methods, how the sources of bias were controlled for, the validity and reliability of the instruments used in the research, data analysis, results, and discussion of a study. The STROBE checklist divides studies into three levels, including weak, moderate, and strong[15]. Studies that have obtained $70 \%$ of the checklist score are included in the study[16] and the scores will present for all researches in the form of a table. Finally, the principles of Preferred Reporting Items of Systematic Reviews (PRISMA) will be followed[17].

- Data analysis:

Data will be analyzed using the STATA software version 11 .

- The type of statistical analysis:

A forest plot will be used to display the results. The sexual violence will be also analyzed based on demographic characteristics. In circumstances that pooling of articles will be deemed inappropriate, authors provide a qualitative discussion of the results.

\section{Results}

This is a systematic review protocol, so the findings are not presented. The results of this study will be submitted for publication after review and extraction from studies. It will also be presented in PROSPERO (International Prospective Register for Systematic Reviews). Registration number is CRD42020191679.

\section{Discussion}

This systematic review studies the sexual violence in perimenopausal women in around the world, the prevalence of sexual violence, the impact of sexual violence on the health of women in perimenopause age, and the demographic factors influencing sexual violence in this age group. According to the improvement in life expectancy and the significant increase in the middle-aged population, and according 
to the world's population transition to old age, it seems to be a greater need for a full understanding of health issues, especially sexual subjects for middle-aged women in particular, sexual issues of this age group. Investigating the studies regarding sexual violence in women in perimenopause can clarify the current situation and identify the underlying factors that affect sexual violence and its adverse outcomes. It is hoped that the results of this research will be a guide for health planners to help to reduce this sociohealth problem and solve the problems of this group of society by providing the necessary counseling programs.

limitations in our study were:

1. The lack of access to gray literature focusing on sexual violence in perimenopausal women.

2. Only articles written in English and Persian will be included

3. If sample size is small, this will limit the quality of the conclusions drawn from this review.

4. Maybe some studied do not meeting the eligibility criteria

\section{List Of Abbreviations}

SDG

sustainable development goals

PRISMA

Preferred Reporting Items of Systematic Reviews

\section{Declarations}

\section{Ethics approval and consent to participate}

The Ethical Committee of Hormozgan University of Medical Sciences, Iran approved this research.

\section{Consent for publication}

The consent form for publication is filled by the authors.

\section{Availability of data and materials}

Not applicable.

\section{Competing interests}

The authors declare no conflict of interests.

\section{Funding}

This study was written without any funding. 


\section{Authors' contributions}

SA and FD considered data gathering approaches and prepared the research proposal under the management of SSM and MH. SA wrote the first draft of the manuscript. SSM contributed the manuscript. FD and $\mathrm{MH}$ edited the final manuscript. All of the authors contributed to the revised and confirmed the final version of the manuscript.

\section{Acknowledgements}

We acknowledged the Hormozgan University of Medical Sciences for providing the research resources.

\section{References}

1. Areskoug-Josefsson $\mathrm{K}$, et al. Education for sexual and reproductive health and rights (SRHR): a mapping of SRHR-related content in higher education in health care, police, law and social work in Sweden. Sex Education. 2019;19(6):720-9.

2. Krug EG, et al. The world report on violence and health. The lancet. 2002;360(9339):1083-8.

3. Pico-Alfonso M, NGarcia-Linares M. The Impact of Physical, Psychological, and Sexual Intimate Male Partner Violence on Women's Mental Health: Depressive Symptoms, Posttraumatic Stress Disorder, State Anxiety, and Suicide. Health s'Women of J. 2006;15(5):599-611.

4. Bahadoran $\mathrm{P}$, et al. The effect of face-to-face or group education during pregnancy on sexual function of couples in Isfahan. Iran J Nurs Midwifery Res. 2015;20(5):582-7.

5. Afshar $\mathrm{M}$, et al. The effect of sex education on the sexual function of women in the first half of pregnancy: a randomized controlled trial. J Caring Sci. 2012;1(4):173-81.

6. Giovanni. Corona, et al, The ESSM Manual of Sexual Medicine, ed. n. edition. 2015, Amsterdam: The European Society for Sexual Medicine (ESSM).

7. Tiwari A, et al. A randomised controlled trial of empowerment training for Chinese abused pregnant women in Hong Kong. BJOG: An International Journal of Obstetrics Gynaecology. 2005;112(9):1249-56.

8. Heidari M, Ghodusi M, Rafiei H. Sexual self-concept and its relationship to depression, stress and anxiety in postmenopausal women. Journal of menopausal medicine. 2017;23(1):42-8.

9. Schwartz B. Sexual assault and the menopause experience. Contemporary OB/GYN. 2019;64(1):389.

10. Shirvani M, Heidari M. Quality of life in postmenopausal female members and non-members of the elderly support association. Journal of menopausal medicine. 2016;22(3):154-60.

11. Cabral P, et al. Influence of menopausal symptoms on sexual function in middle-aged women. Rev Bras Ginecol Obstet. 2012;34(7):329-34.

12. Bows H. Sexual violence against older people: A review of the empirical literature. Trauma Violence Abuse. 2018;19(5):567-83. 
13. Luoma M-L, et al., Prevalence study of abuse and violence against older women: results of a multicultural survey conducted in Austria, Belgium, Finland, Lithuania, and Portugal. 2011, National Institute for Health and Welfare.

14. Gibson C, et al. Associations of Intimate Partner Violence, Sexual Assault, and Posttraumatic Stress Disorder With Menopause Symptoms Among Midlife and Older Women. JAMA Internal Medicine. 2019;179(1):80.

15. Vandenbroucke J, et al. Strobe Initiative Strengthening the Reporting of Observational Studies in Epidemiology (STROBE): explanation and elaboration. PLoS Med. 2007;4(10):297.

16. Cheraghi Z, Irani AD, Rezaeian S. Quality of Cohort Studies Reporting Post the Strengthening the Reporting of Observational Studies in Epidemiology (STROBE) Statement Jalal Poorolajal. Epidemiol Health, 2011. 33.

17. Adams AD, et al. Use of the STROBE checklist to evaluate the reporting quality of observational research in obstetrics. Obstetrics Gynecology. 2018;132(2):507-12. 